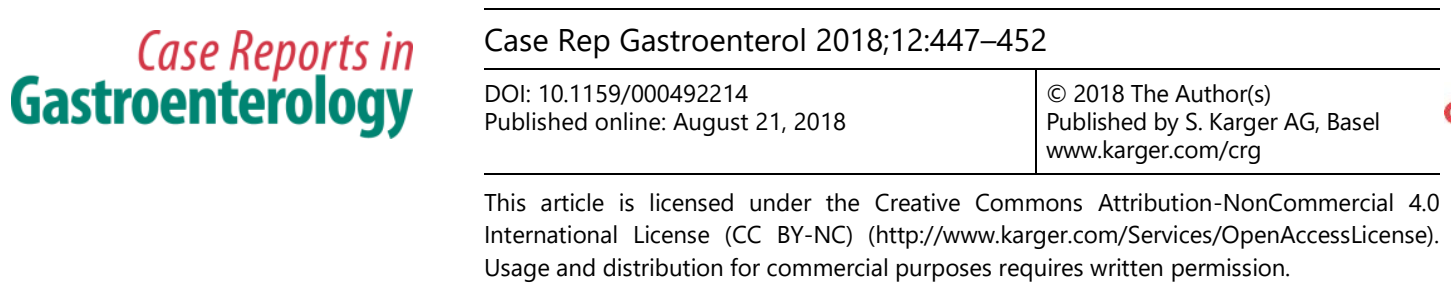

\title{
Drug-Induced Autoimmune Hepatitis in a Patient Treated with Minocycline: A Rare Adverse Effect
}

\author{
Jamil Shah $^{\mathrm{a}}$ Abul Shahidullah ${ }^{\mathrm{b}}$ Yingxian Liuc \\ aDepartment of Internal Medicine, The Brooklyn Hospital Center, Clinical Affiliate of \\ The Mount Sinai Hospital, Academic Affiliate of The Icahn School of Medicine at Mount \\ Sinai, Brooklyn, NY, USA; ${ }^{b}$ Department of Medicine, Henry J. Carter Specialty Hospital and \\ Nursing Facility, New York, NY, USA; 'Department of Anatomic Pathology, The Brooklyn \\ Hospital Center, Clinical Affiliate of The Mount Sinai Hospital, Academic Affiliate of \\ The Icahn School of Medicine at Mount Sinai, Brooklyn, NY, USA
}

\section{Keywords}

Minocycline $\cdot$ Drug-induced liver injury · Drug-induced hepatitis · Autoimmune hepatitis

\begin{abstract}
Drug-induced autoimmune hepatitis is an acute and potentially severe adverse effect, which has been reported following the long-term use of minocycline. The condition's typical biochemical findings include an elevated antinuclear antibody titer, hypergammaglobulinemia with elevated levels of serum immunoglobulin $\mathrm{G}$, and, sometimes, positive anti-smooth muscle antibodies. Characteristically, transaminase levels are very elevated, while markers of cholestasis and bilirubin levels are mildly increased, and histological features are very similar to those observed in sporadic autoimmune hepatitis. Here, we describe an interesting case of a young female who developed drug-induced autoimmune hepatitis after taking minocycline for the treatment of acne vulgaris.

(C) 2018 The Author(s)

Published by S. Karger AG, Basel
\end{abstract}

\section{Introduction}

Minocycline is an oral broad-spectrum tetracycline antibiotic that is used to treat moderate to severe acne vulgaris as well as certain sexually transmitted infections. Adverse effects 


\section{Case Reports in Gastroenterology}

Case Rep Gastroenterol 2018;12:447-452 DOI: $10.1159 / 000492214$

(c) 2018 The Author(s). Published by S. Karger AG, Basel www.karger.com/crg

Shah et al.: Drug-Induced Autoimmune Hepatitis in a Patient Treated with Minocycline: A Rare Adverse Effect

of the medication are uncommon but include autoimmune syndromes, such as autoimmune hepatitis, drug-induced lupus, vasculitis, and serum sickness [1]. Although the autoimmunelike hepatitis triggered by minocycline varies in severity, cases of fulminant hepatitis as well as some patients requiring corticosteroid therapy have been reported [2]. The medication induces a hepatocellular pattern of liver injury that mimics sporadic autoimmune hepatitis [3]. However, minocycline-induced autoimmune hepatitis can be differentiated from "classic" autoimmune hepatitis by the patient's swift recovery after discontinuing the offending drug and no relapse of the condition upon discontinuation of treatment with corticosteroids [4].

\section{Case Presentation}

A 31-year-old Chinese female with a past medical history of acne vulgaris presented to the emergency department with nausea, vomiting, and abdominal pain for the last 3-4 days. She had experienced 3 episodes of non-bloody, non-bilious vomiting. She described a persistent, dull, aching pain in the right upper quadrant of the abdomen that had been increasing in intensity, particularly over the last 2 days. She denied any recent change in her diet. She reported that no one in her household had experienced similar symptoms. She denied any recent symptoms of fevers, chills, diarrhea, constipation, blood in the stool, black stools, change in appetite, weight loss, or any other symptoms.

The patient had immigrated to the United States from China 3 months earlier. She reported no previous history of alcohol consumption, intravenous drug use, acquiring tattoos or nonsterile piercings, receiving transfusions of blood or blood products, sexual promiscuity, occupational exposure to toxins (she worked as a cashier in a grocery store), or prior liver diseases. She reported no family history of liver diseases. Furthermore, she denied any drug allergies.

Upon medication reconciliation, it was discovered that the patient had been taking minocycline, on her own and not under medical supervision, for the past 3 months. She had been taking it to treat severe acne vulgaris, and she had brought it with her from China. Attributing her vomiting and worsening abdominal pain to the medication, she had discontinued it 2 days earlier. She denied taking any other medications nor any complementary, alternative, or herbal medicines.

The patient was afebrile (temperature $99.0^{\circ} \mathrm{F}$ ) and was hemodynamically stable (pulse rate 76 beats/min, blood pressure 142/70 $\mathrm{mm} \mathrm{Hg}$ ). On physical examination, the abdomen was soft and not distended. There was mild tenderness in the right upper quadrant, the liver edge was palpable, and normoactive bowel sounds were present. Murphy's sign was negative, and there was no rebound tenderness, guarding, or rigidity.

Initial laboratory testing was remarkable for hypokalemia with a serum potassium of 3.1 $\mathrm{mEq} / \mathrm{L}$ (range 3.5-5.1). The rest of the serum electrolytes and the complete blood count were within normal limits. Significantly elevated transaminase levels with aspartate aminotransferase of $718 \mathrm{U} / \mathrm{L}$ (range 10-35) and alanine aminotransferase of $1138 \mathrm{U} / \mathrm{L}$ (range 10-55) were also found. There were mildly increased total bilirubin of $1.8 \mathrm{mg} / \mathrm{dL}$ (normal value $<1.3$ ) and mildly increased direct bilirubin of $0.8 \mathrm{mg} / \mathrm{dL}$ (normal value $<0.6$ ). Alkaline phosphatase was $160 \mathrm{U} / \mathrm{L}$ (range 40-150), Gamma-glutamyltransferase was $112 \mathrm{U} / \mathrm{L}$ (range 5-50), serum total protein was $8.4 \mathrm{~g} / \mathrm{dL}$ (range 6.0-8.3), serum albumin was $3.6 \mathrm{~g} / \mathrm{dL}$ (range 3.5-5.5), and the international normalized ratio was 1.9 (range 0.8-1.2). Prior liver function tests (LFTs), before she had started taking minocycline, were not available. The patient's Model for EndStage Liver Disease score was calculated to be 18 upon arrival. 


\section{Case Reports in Gastroenterology}

Case Rep Gastroenterol 2018;12:447-452 DOI: $10.1159 / 000492214$

(c) 2018 The Author(s). Published by S. Karger AG, Basel www.karger.com/crg

Shah et al.: Drug-Induced Autoimmune Hepatitis in a Patient Treated with Minocycline: A Rare Adverse Effect

Further laboratory testing showed that the serum iron level, total iron-binding capacity, serum ferritin, ceruloplasmin level, and thyroid function tests were within normal limits. An acetaminophen level was negative. Viral serologies for hepatitis B virus, hepatitis C virus, cytomegalovirus, and Epstein-Barr virus were negative. However, an autoimmune workup revealed an elevated antinuclear antibody (ANA) titer (1:160; normal value <1:40), positive anti-smooth muscle antibodies, and the finding of hypergammaglobulinemia $(3.2 \mathrm{~g} / \mathrm{dL}$; range 0.7-1.6) with elevated levels of serum immunoglobulin G $(3,176 \mathrm{mg} / \mathrm{dL}$; range $700-$ $1,600)$. An abdominal ultrasound revealed mild hepatomegaly with increased liver echogenicity (Fig. 1).

The patient's vomiting improved with antiemetic agents, her abdominal pain improved with analgesic agents, and her serum potassium was repleted to normal. The bilirubin levels decreased steadily from day 1 of the hospital admission, but the transaminase levels continued to rise despite discontinuation of minocycline (Fig. 2). On day 7, she was started on the treatment with prednisolone at an initial dose of $50 \mathrm{mg} /$ day.

Furthermore, an interventional radiology-guided liver biopsy was performed for confirmation of the diagnosis as well as to assess the degree of liver injury (Fig. 3).

After 2 days of treatment with prednisolone, the patient's transaminase levels began to decrease steadily, ultimately falling to aspartate aminotransferase $45 \mathrm{U} / \mathrm{L}$ and alanine aminotransferase $69 \mathrm{U} / \mathrm{L}$ (Fig. 2). Prednisolone was continued for 4 weeks, and the LFTs were within normal range at a follow-up visit 1 month later. The patient made a full recovery.

\section{Discussion}

Drug-induced hepatitis can occur after the use of a number of medications (both prescription and over-the-counter), herbal supplements, and dietary supplements. It can be described in a few ways: the biochemical presentation (hepatocellular injury, cholestatic injury, or a mixed pattern with features of both types of injury); the mechanism of hepatotoxicity (either in a dose-dependent, predictable manner or in an unpredictable, idiosyncratic way); and the histologic findings (hepatitis, cholestasis, or steatosis) [5]. Typically, drug-induced hepatitis is initially defined by its clinical presentation and laboratory data. It can then be further described by histologic findings, if a liver biopsy is performed to confirm the diagnosis or to assess the degree of liver injury.

The autoimmune-like hepatitis that is induced by minocycline can mimic sporadic autoimmune hepatitis [6-8]. For instance, both diseases can present either with acute hepatitis or insidiously. Moreover, the autoantibody profiles are similar with nearly universal existence of ANA in minocycline-induced autoimmune hepatitis and elevated frequencies of ANA and antismooth muscle antibodies in classic, or type 1, autoimmune hepatitis [6-8]. With regard to treatment, patients with minocycline-induced autoimmune hepatitis without jaundice can frequently be managed by simply withdrawing the medication. Those patients with severe hepatitis often require corticosteroid therapy but, even so, only for a limited period of 2-4 months. In contrast, in patients with classic autoimmune hepatitis, immunosuppressive therapy is recommended for at least 18 months, once remission has been established [9].

Physicians should be informed about and suspect drug-induced hepatitis for any individual taking minocycline who presents with elevated LFTs. In particular, internists and dermatologists who prescribe this medication to treat acne vulgaris should be aware of this potentially severe adverse effect and discontinue it immediately when suspicion is raised. Thus, when prescribing minocycline, the LFTs should be carefully watched. The way to make the 
diagnosis is discontinuing the medication followed by improvement in LFTs. Moreover, before establishing the diagnosis of sporadic autoimmune hepatitis, a recent intake of minocycline should be ruled out. When stopping the offending agent alone does not lead to improvement in LFTs and the patient's clinical status, a thorough history should be taken, and initial diagnostic testing should be completed to rule out additional etiologies. If they are negative, a liver biopsy and/or treatment with corticosteroids should be considered.

\section{Statement of Ethics}

The authors have no ethical conflicts to disclose.

\section{Disclosure Statement}

The authors of this case report declare that neither financial support nor grant support has been received for the preparation of this paper. The authors declare that they have no competing interests and no affiliations with or involvement in any organization or entity with financial or nonfinancial interests (such as personal or professional relationships, affiliations, knowledge, or beliefs) in the subject matter discussed in this paper.

\section{References}

1 Elkayam 0, Yaron M, Caspi D. Minocycline-induced autoimmune syndromes: an overview. Semin Arthritis Rheum. 1999 Jun;28(6):392-7.

2 Lawrenson RA, Seaman HE, Sundström A, Williams TJ, Farmer RD. Liver damage associated with minocycline use in acne: a systematic review of the published literature and pharmacovigilance data. Drug Saf. 2000 Oct;23(4):333-49.

3 Björnsson E, Talwalkar J, Treeprasertsuk S, Kamath PS, Takahashi N, Sanderson S et al. Drug-induced autoimmune hepatitis: clinical characteristics and prognosis. Hepatology. 2010 Jun;51(6):2040-8.

4 Aldenhoven M, van Enk JG, Avis WA. [Minocycline-induced autoimmune hepatitis]. Ned Tijdschr Geneeskd. 2013;157(3):A5465.

5 Chang CY, Schiano TD. Review article: drug hepatotoxicity. Aliment Pharmacol Ther. 2007 May;25(10):1135-51.

6 Krawitt EL. Autoimmune hepatitis. N Engl J Med. 2006 Jan;354(1):54-66.

7 Teitelbaum JE, Perez-Atayde AR, Cohen M, Bousvaros A, Jonas MM. Minocycline-related autoimmune hepatitis: case series and literature review. Arch Pediatr Adolesc Med. 1998 Nov;152(11):1132-6.

8 Goldstein NS, Bayati N, Silverman AL, Gordon SC. Minocycline as a cause of drug-induced autoimmune hepatitis. Report of four cases and comparison with autoimmune hepatitis. Am J Clin Pathol. 2000 Oct;114(4):591-8.

9 Healy J, Alexander B, Eapen C, Roberts-Thomson IC. Minocycline-induced autoimmune hepatitis. Intern Med J. 2009 Jul;39(7):487-8. 


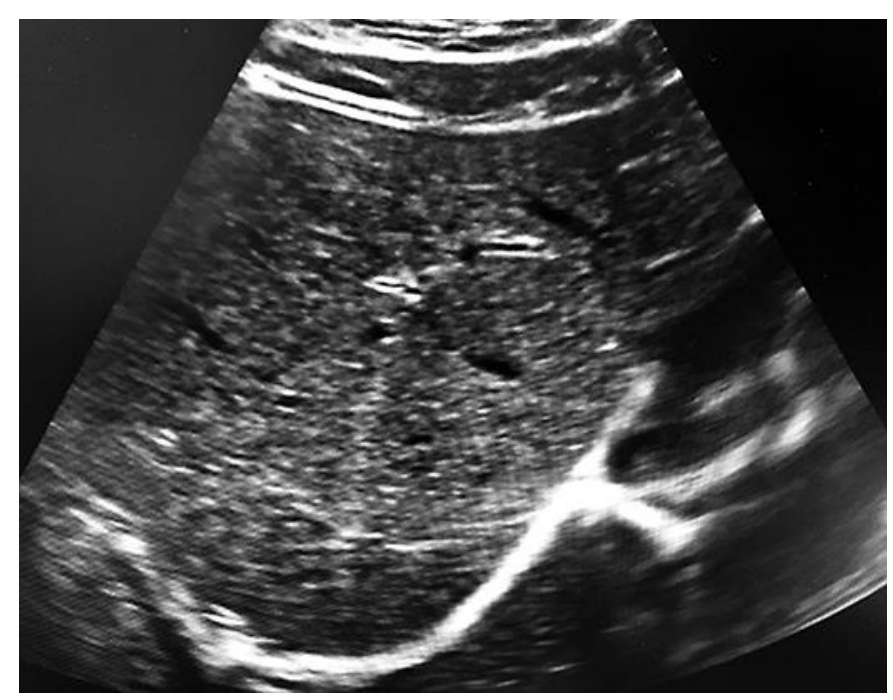

Fig. 1. Abdominal ultrasound. There is mild hepatomegaly with increased liver echogenicity.

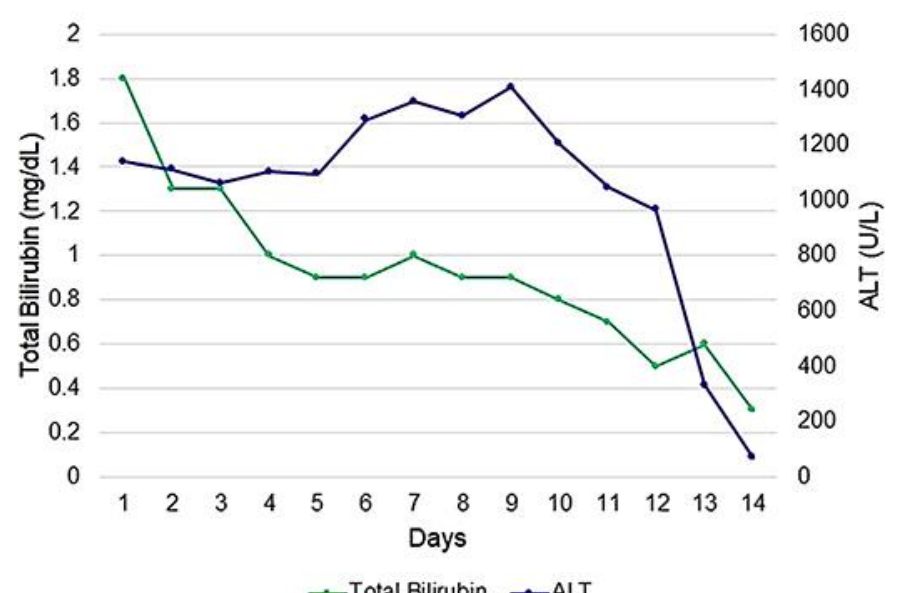

Fig. 2. Changes in total bilirubin and alanine aminotransferase before and after treatment with prednisolone. Prednisolone, at an initial dose of $50 \mathrm{mg} /$ day, was started on day 7 of the hospital admission. 


\section{Case Reports in Gastroenterology}

\begin{tabular}{l|l}
\hline Case Rep Gastroenterol 2018;12:447-452 \\
\hline DOI: 10.1159/000492214 & $\begin{array}{l}\text { c } 2018 \text { The Author(s). Published by S. Karger AG, Basel } \\
\text { www.karger.com/crg }\end{array}$ \\
\hline
\end{tabular}

Shah et al.: Drug-Induced Autoimmune Hepatitis in a Patient Treated with Minocycline: A Rare Adverse Effect
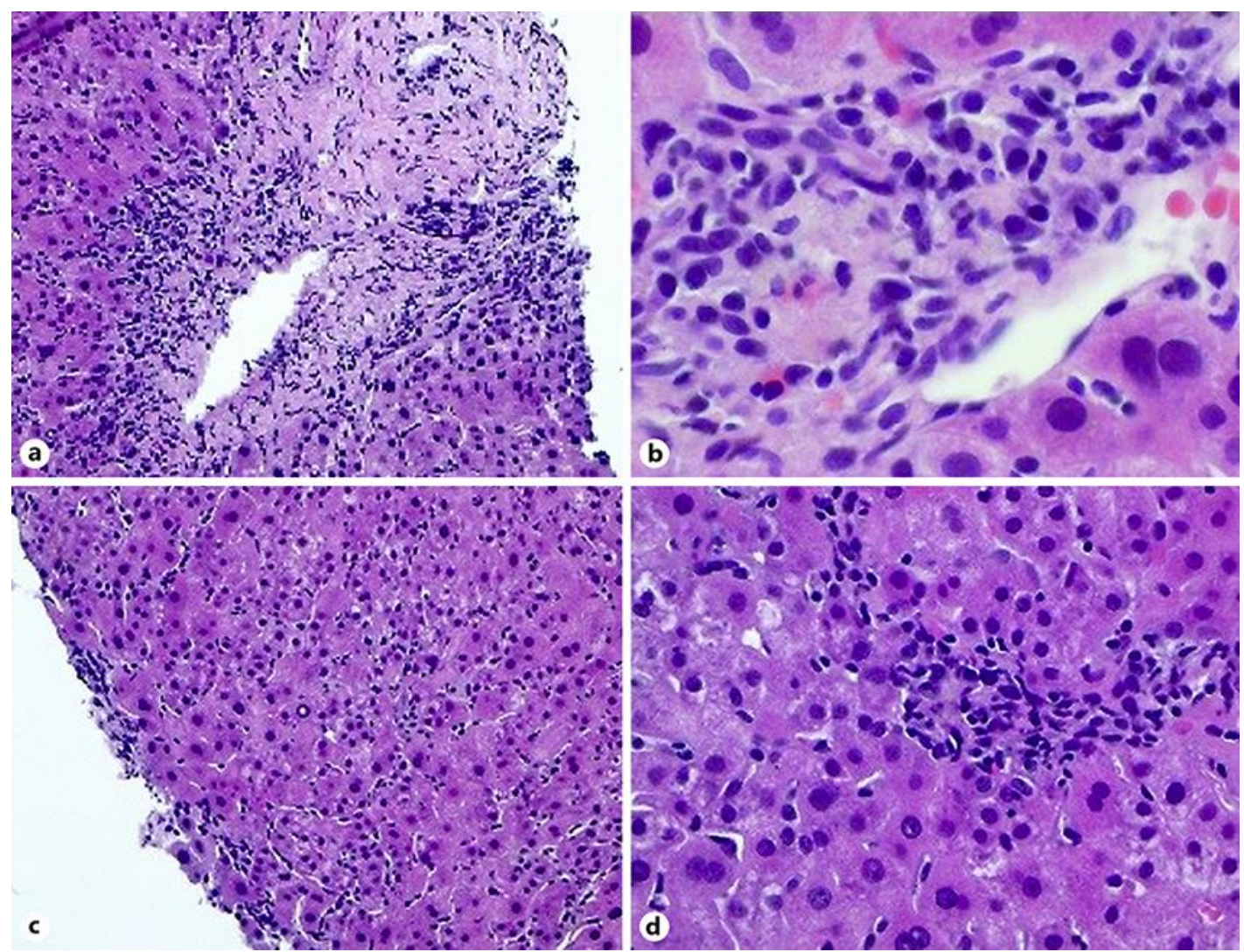

Fig. 3. a-d Histopathologic images of the patient's drug-induced hepatitis from liver biopsy. a Piecemeal necrosis. There is chronic inflammation in the portal tracts. H\&E stain. $\times 100$. $\mathbf{b}$ Again, there is chronic inflammation in the portal tracts with many plasma cells, lymphocytes, and occasional eosinophils. H\&E stain. $\times 400$. c, $\mathbf{d}$ Spotty necrosis. There is mild chronic inflammation in the portal tracts, as well as ballooning degeneration of hepatocytes indicative of hepatocellular injury. H\&E stain. $\times 100$ and $\times 400$, respectively. 